\title{
Fabrication and measurement of optical waveguide sensor based on localized surface plasmon resonance
}

\author{
Hyeong-Min Kim, Jae-Hyoung Park ${ }^{*}$ and Seung-Ki Lee*
}

\begin{abstract}
We propose a novel localized surface plasmon resonance (LSPR) sensor system based on polymer material. The proposed LSPR system consists of the incident medium with low-loss polymer waveguide and the chemically immobilized plasmonic nanoparticles for on-chip LSPR sensing. Because of low coupling efficiency of conventional methods, usually intricate test equipment such as dark field microscopes equipped with cooled charge-coupled device detectors is required to perform nanoparticles LSPR sensing. Using a polymer optical waveguide instead of conventional free-space excitation techniques (e.g., using an objective lens) in the LSPR sensing system offers miniaturization, low cost, and potable sensing capability. The integration of this hybrid plasmonic-photonic sensor with optical system based on fiber optic is measured to refractive index with a sensitivity of 3.10/RIU.
\end{abstract}

Keywords: Localized surface plasmon resonance, Optical waveguide, Au nanoparticle, Optical fiber, Miniaturization

\section{Introduction}

The oscillations of free electrons in noble metal nanoparticles when the light is incident is called localized surface plasmon (LSP), and the phenomenon that the scattered signals in incident lights by nanoparticles are amplified with LSP is referred to localized surface plasmon resonance (LSPR) [1, 2]. One of the most remarkable effects of LSPR is that the intensity of LSPR is sensitive to changes of the refractive indices around noble metal nanoparticles $[3,4]$. The advantages of LSPR sensors having this characteristic are real-time analysis, label-free detection, and high sensitivities [5-7]. However, the most of LSPR sensors have been developed to method that the incident lights are focused to nanoparticles in free-space by the objective lens, which limits the coupling efficiency, miniaturization, cost-effectiveness, and accessibility [8-12].

In this study, we propose a novel LSPR based optical waveguide (OWG) sensor which is fabricated by SU-8 photoresist (PR) through relatively simple process that

\footnotetext{
*Correspondence: parkjae@dankook.ac.kr; skilee@dankook.ac.kr Department of Electronics and Electrical Engineering, Dankook University, Yongin 16890, Republic of Korea
}

can efficiently combine the light with nanoparticles. A simple optical measurement set-up is also constructed by applying the optical fiber, and the proposed sensor is used to LSPR signal measurement according to the refractive index changes of the surrounding medium. The LSPR based OWG sensor is expected to the application as biosensor with advantages in miniaturization, low cost, and portability in the future.

\section{Materials and methods \\ Design and fabrication of OWG}

The OWG of stair-shape is designed as shown in Fig. 1a because the situation that the lights directly enter the detector from the light source without the pass of OWG can cause the noise in the observation of LSPR signal. Considering the fact that light transmission loss of $0.1 \mathrm{~dB}$ per $1 \mathrm{~mm}$ occurs in the OWG using SU-8 (2075, Microchem, Newton, MA, USA), the distance between both optical fiber holders is fabricated as short as possible $2 \mathrm{~mm}$ [13]. If the stair angle $(\theta)$ of OWG becomes large, the light transmission efficiency is decreased due to the increases of the bending angle and the effective length of OWG [14, 15]. On the other hand, when $\theta$ becomes smaller, the light from optical fiber connected to the 


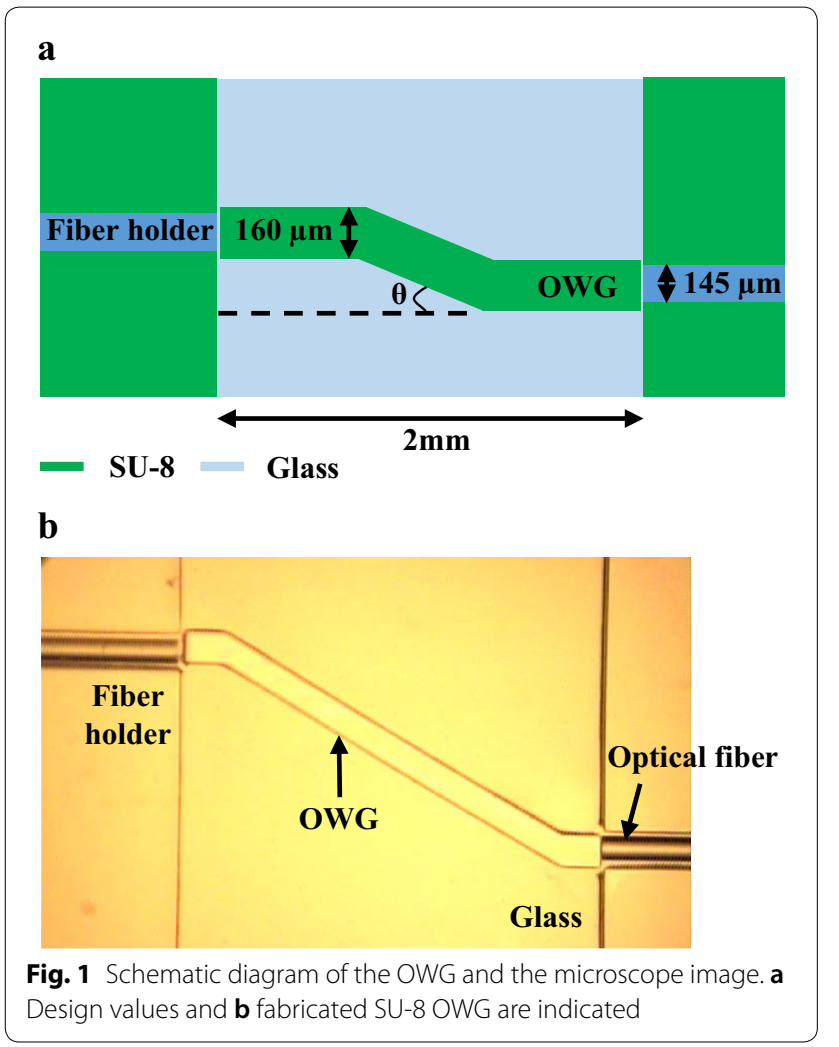

source can be directly incident on the opposite optical fiber, which makes it difficult to observe the LSPR signal. In order to improve the light transmission efficiency of OWG, the $\theta$ is selected to $30^{\circ}$ within a range in which the effective length of OWG is shortened and the light is not directly transmitted between the optical fibers. The width of OWG is planned to be $160 \mu \mathrm{m}$, considering that the coupled optical fiber diameter which includes core and cladding is $125 \mu \mathrm{m}$ and the spread of light emitted from the optical fiber. Finally, the width of optical fiber holder is decided to be $145 \mu \mathrm{m}$ to allow enough area for the optical fiber. A gap of about $10 \mu \mathrm{m}$ is set on both sides of the optical fiber.

The OWG was simply fabricated through a single lithography process (MDA-400M, Midas system, Daejeon, Republic of Korea) of SU-8. The excellent optical transparency beyond $400 \mathrm{~nm}$ and the low transmission loss of SU-8 make it a preferred material for the fabrication of various optical components and systems such as OWGs, splitters, directional couplers, and gratings [16-18]. SU-8 has a high refractive index of 1.59 at the wavelength of $640 \mathrm{~nm}$ used in the measurement, which can increase the light guiding efficiency due to the large contrast of the refractive indices between $\mathrm{SU}-8$ and organic-based tetraethyl orthosilicate (TEOS) used as the cladding [19]. The refractive index of TEOS is 1.38 .
SU-8 also has good mechanical and thermal stability, and is suitable for biosensing applications due to its biocompatibility and chemically stable characteristic [20, 21]. The SU-8 was spin-coated (ACE-200, Dong ah, Seoul, Republic of Korea) on 4 in. glass wafer (Boro33, DS semicon, Anyang-si, Republic of Korea) at $1700 \mathrm{rpm}$ for $40 \mathrm{~s}$. At this time, the soft bake was sequentially performed at $65{ }^{\circ} \mathrm{C}$ for $20 \mathrm{~min}$ and at $95{ }^{\circ} \mathrm{C}$ for $55 \mathrm{~min}$, and the exposure was continued for $60 \mathrm{~s}$ at intensity of $25 \mathrm{~mW} / \mathrm{cm}^{2}$. The post exposure bake was carried out at $65{ }^{\circ} \mathrm{C}$ and $95{ }^{\circ} \mathrm{C}$ for $10 \mathrm{~min}$ and $30 \mathrm{~min}$, respectively. Finally, the sample was developed in AZ 1500 thinner (AZ electronic materials, Seoul, Republic of Korea) during $90 \mathrm{~min}$ to complete the OWG fabrication. Figure $1 \mathrm{~b}$ is micrograph when the optical fibers (FG105LCA, Thorlabs, Newton, NJ, USA) are fixed to the fabricated fiber holder. The optical fiber was immobilized to the fiber holder using ultraviolet curable resin (NOA61, Norland, Cranbury, NJ, USA). Figure 2 shows the field emission scanning electron microscopy (FE-SEM, S-4800, Hitachi, Ibaraki, Japan) images of OWG and optical fiber holder. The width and height of fabricated OWG were measured to be $162.4 \mu \mathrm{m}$ and $126.3 \mu \mathrm{m}$, respectively. The width of the fiber holder was also $144.5 \mu \mathrm{m}$.

\section{Fabrication of OWG sensor based on LSPR}

The method to immobilize the $\mathrm{Au}$ nanoparticles on OWG using SU-8 consists of four steps. In the first step, the oxygen plasma (CUTE, Femto science, Yongin-si, Republic of Korea) was treated on OWG with $60 \mathrm{~W}$ power and $0.8 \mathrm{~m}$ Torr during $50 \mathrm{~s}$. The oxygen plasma treatment removes the organic compound and functionalizes the surface of OWG with hydroxyl group [22, 23]. Next, the sample was reacted with $1 \%$ TEOS $(98 \%$, Sigma aldrich, St. Louis, MO, USA) solution in isopropanol (99.5\%, Daejung, Siheung-si, Republic of Korea) for $3 \mathrm{~h}$, which acts as the cladding in OWG and controls the Au nanoparticles density on the surface [24]. In the third step, 1\% 3-(ethoxydimethylsilyl)-propylamine (97\%, Sigma aldrich) solution based on isopropanol was coated on the OWG for $2 \mathrm{~h}$ to form the self-assembled monolayers which have the amine groups with positive charges to immobilize the $\mathrm{Au}$ nanoparticles on the OWG surface. Finally, the Au nanoparticles which are prepared at about $47.8 \mathrm{~nm}$ diameter were attached on OWG during $6 \mathrm{~h}$. The $\mathrm{Au}$ nanoparticles were synthesized through the Turkevich method by reducing the $\mathrm{Au}(\mathrm{III})$ chloride trihydrate (99.9\%, Sigma aldrich) using sodium citrate dehydrate (99\%, Daejung) [25, 26]. Since the Au ions are reduced by citrate, the negative charges are present on the surface of the Au nanoparticles, which enables the electrostatic bonding with the positive charges of amine groups on the OWG $[27,28]$. The Au nanoparticles are immobilized on 


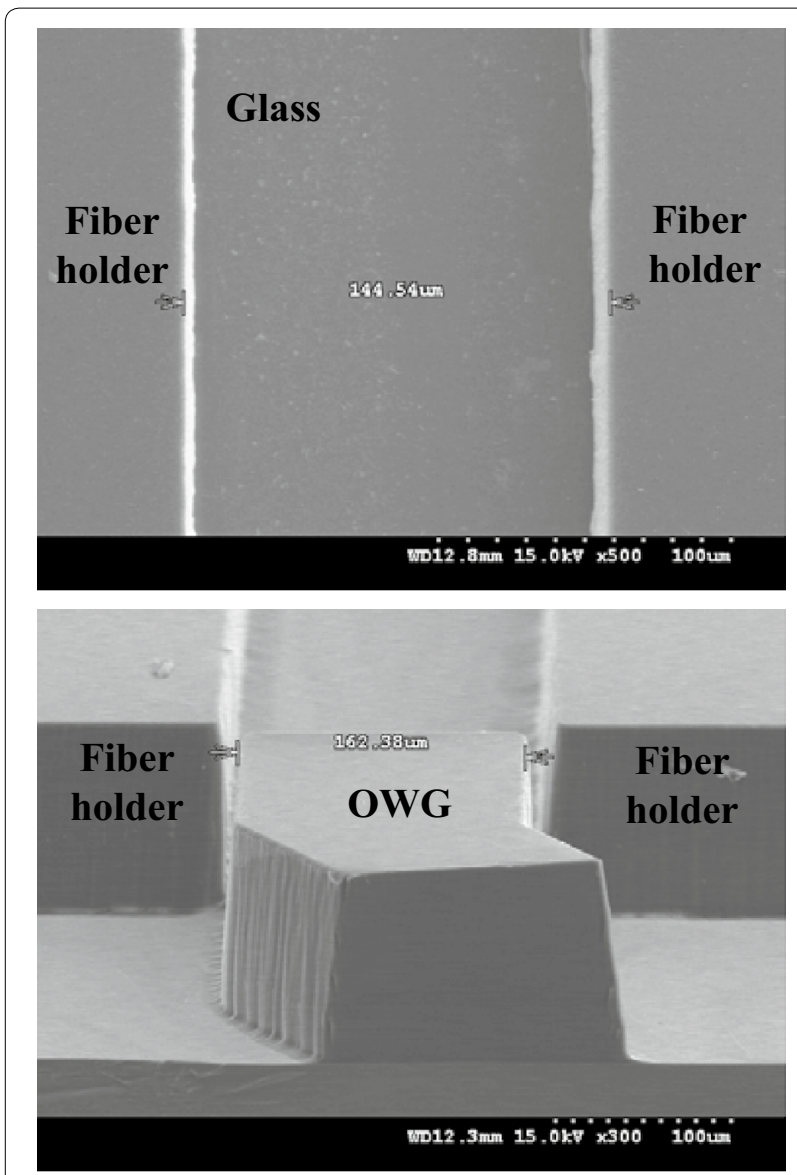

Fig. 2 The FE-SEM photograph of fabricated OWG the side surface as well as the top surface of OWG. However, the nanoparticles are not attached between OWG and optical fiber in order to prevent the decrease of coupling efficiency. When Au nanoparticles are immobilized on the gap between OWG and fiber, the incident light from optical fiber will be reflected back to the fiber by the $\mathrm{Au}$ nanoparticles. The fabrication process of OWG sensor based on LSPR and the FE-SEM photography at the surface of proposed sensor were displayed in Fig. 3. On the OWG surface, the Au nanoparticles were mostly present as monomer without excessive aggregation.

\section{Results and discussion}

\section{Optical measurement set-up}

The optical measurement set-up for the record of LSPR signal is simply composed as shown in Fig. 4. The light from the laser (Iflex-2000, Qioptiq, Hamble-le-rice, UK) is transmitted to the OWG sensor through the optical fiber, which reacts with the Au nanoparticles on the sensor surface. Then, the reacted lights are again collected through the optical fiber to the photodetector (PDA36A, Thorlabs). The collected signals by the photodetector are sent to a computer by the data acquisition (USB-6210, National instruments, Austin, TX, USA) and observed in real-time. In this set-up, the optical fibers are positioned as close to OWG as possible [29].

\section{Refractive index measurement using the OWG sensor} based on LSPR

It is evaluated that the OWG sensor generates the changed LSPR intensities according to the increase of

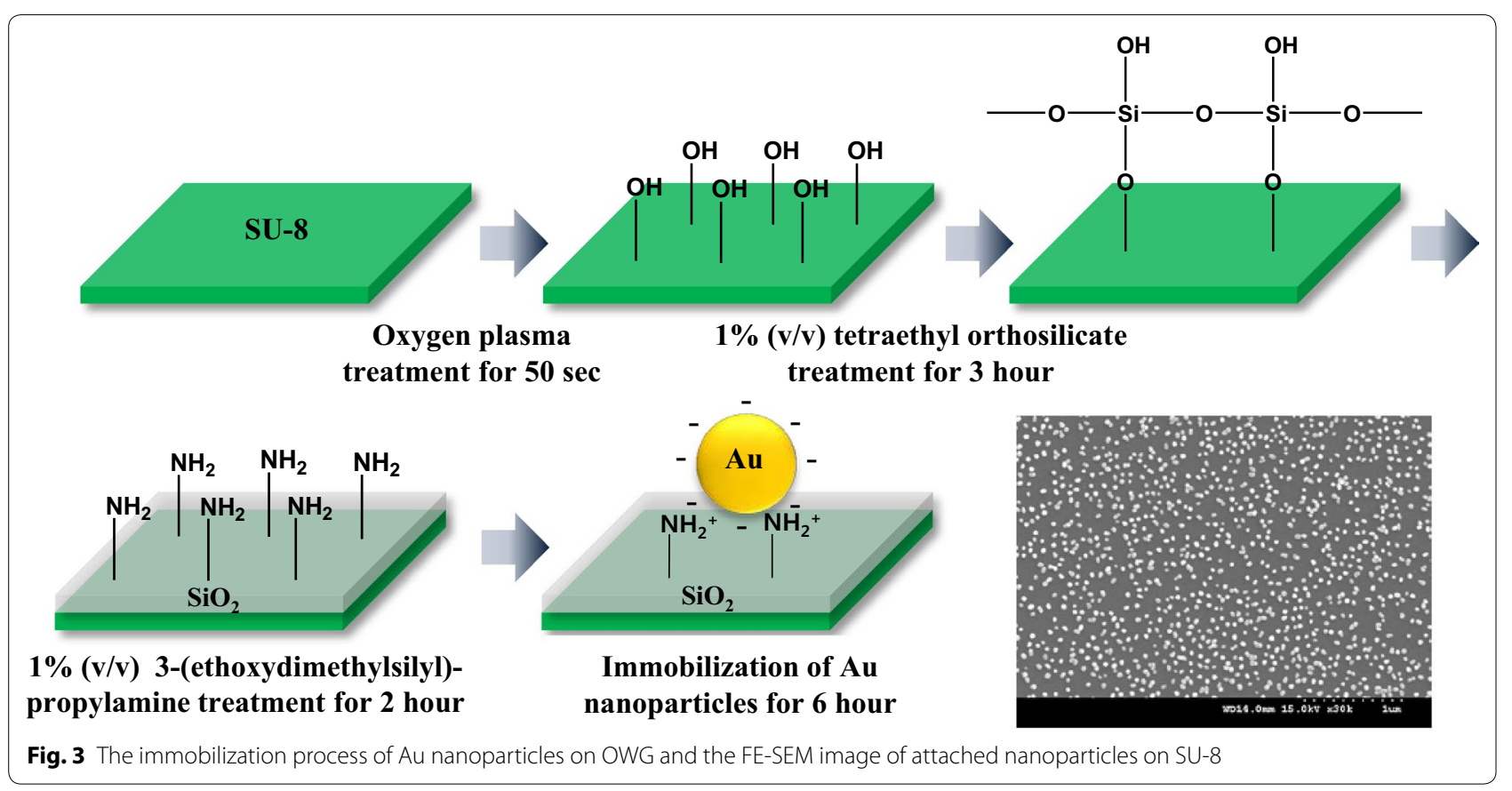




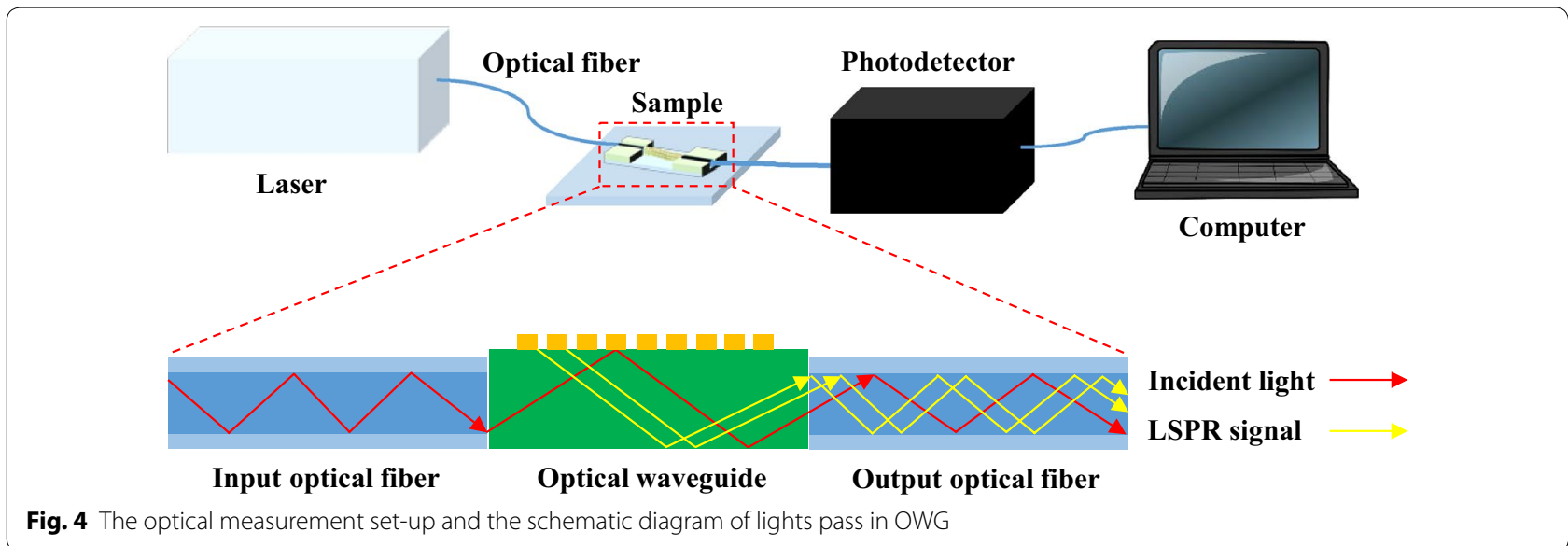

refractive index around $\mathrm{Au}$ nanoparticles. When six refractive index solutions with a spacing of 0.01 from 1.33 to 1.38 were sequentially supplied to the sensor, the LSPR intensities were gradually increased in Fig. 5a. The refractive index sensitivity which is defined by the normalized intensity change per refractive index unit (RIU) and the coefficient of determination $\left(\mathrm{R}^{2}\right)$ indicating the degree of correlation between the two variables are shown in Fig. 5b [30, 31]. The sensitivity of proposed sensor was $3.10 / \mathrm{RIU}$ and the $\mathrm{R}^{2}$ was 0.99 , which mean the high linearity between the refractive index and the output of OWG sensor. The normalized intensity is used to calculate the sensitivity because the LSPR intensity depends on external factors such as the laser power, the gain of photodetector, and the alignment between light source and fiber [32]. The normalized intensity is defined as the relative signal based on the measured intensity at $1.33[33]$.

\section{Conclusion}

The conventional methods that the light exposes into a free space during LSPR measurement have been problematic such as the insufficient optical coupling efficiency and the requirement of expert skill for the alignment of light. To solve these problems, we applied the OWG that do not expose the light to the air and suggested using it as an LSPR sensor. The low-loss OWG was fabricated on glass wafer by SU-8 using a single lithography process and the OWG sensor based on LSPR is completed by immobilizing the Au nanoparticles on OWG. In addition, it was possible to construct the compact optical system by using optical fiber, which enables the simple transmission and collection of light. The proposed sensor was applied as a refractive index sensor and it was confirmed that the OWG sensor based on LSPR exhibits a very linear response with the change of refractive index. Based
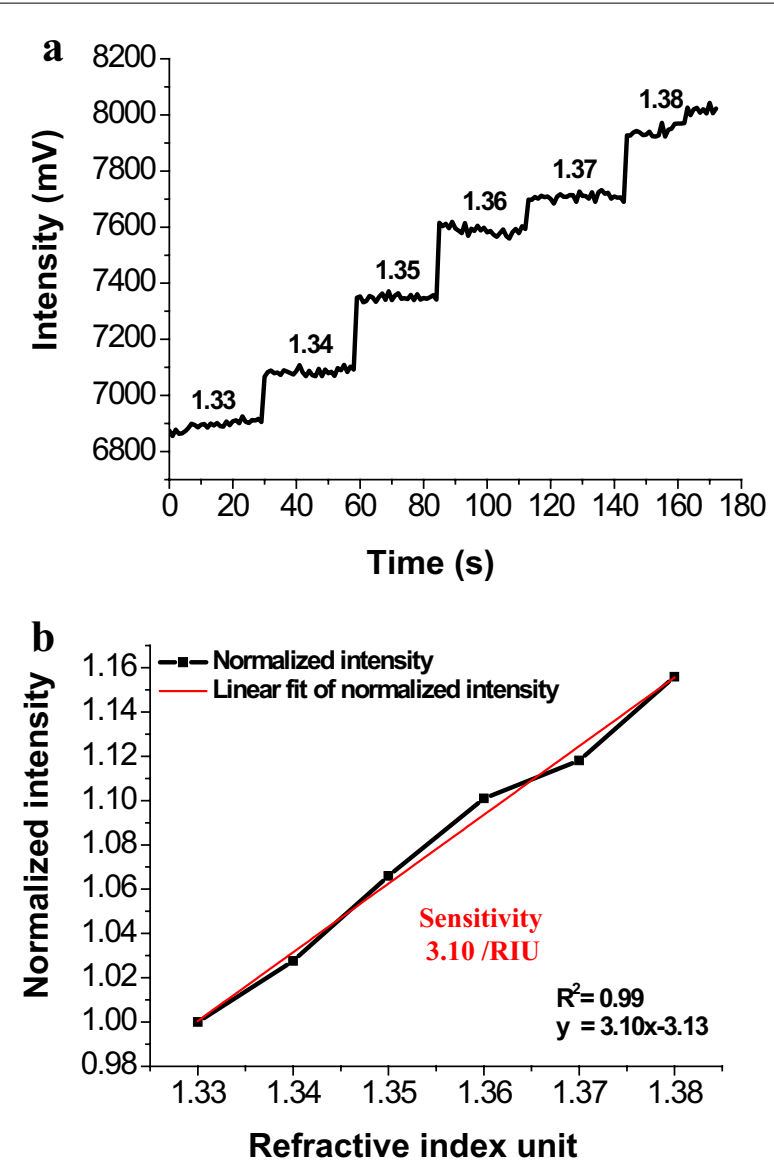

Fig. 5 The measurement result. a LSPR intensity changes according to increase of refractive indices. $\mathbf{b}$ The amount of increased intensity per refractive index unit. The intensities are normalized as reference to the signal at 1.33 
on these results, if a number of OWGs are integrated into a single chip, it is expected that the various target molecules can be multi measured in real-time as the biosensor application.

\section{Acknowledgements \\ Not applicable.}

\section{Authors' contributions}

SKL and JHP devised the idea and supervised the project. SKL, JHP, and HMK discussed the design and experimental setup. HMK performed the experiment and drafted the manuscript. All authors edited the manuscript. All authors read and approved the final manuscript.

\section{Funding}

This work was supported by the National Research Foundation of Korea (NRF) grant funded by the Korea government (MSIT) (No. NRF-2018R1A2B6001361).

\section{Availability of data and materials}

The datasets supporting the conclusions of this article are included within the article.

\section{Competing interests}

The authors declare that they have no competing interests.

Received: 23 April 2019 Accepted: 7 June 2019

Published online: 26 June 2019

\section{References}

1. Langer J, Novikov SM, Liz-Marzan LM (2015) Sensing using plasmonic nanostructures and nanoparticles. Nanotechnology 26:322001

2. Manzano M, Vizzini P, Jia K, Adam PM, lonescu RE (2016) Development of localized surface plasmon resonance biosensors for the detection of Brettanomyces bruxellensis in wine. Sens Actuators B Chem 223:295-300

3. Dahlin AB, Tegenfeldt JO, Höök F (2006) Improving the instrumental resolution of sensors based on localized surface plasmon resonance. Anal Chem 78:4416-4426

4. Kim HM, Nam KT, Lee SK, Park JH (2018) Fabrication and measurement of microtip-array-based LSPR sensor using bundle fiber. Sens Actuatiors A Phys 271:146-152

5. Srivastava SK, Verma RK, Gupta BD (2009) Theoretical modeling of a localized surface plasmon resonance based intensity modulated fiber optic refractive index sensor. Appl Opt 48:3796-3802

6. Jeong HH, Erdene N, Park JH, Jeong DH, Lee HY, Lee SK (2013) Real-time label-free immunoassay of interferon-gamma and prostate-specific antigen using a fiber-optic localized surface plasmon resonance sensor. Biosens Bioelectron 39:346-351

7. Xie L, Yan X, Du Y (2014) An aptamer based wall-less LSPR array chip for label-free and high throughput detection of biomolecules. Biosens Bioelectron 53:58-64

8. McFarland AD, Van Duyne RP (2003) Single silver nanoparticles as realtime optical sensors with zeptomole sensitivity. Nano Lett 3:1057-1062

9. Xiang G, Zhang N, Zhou X (2010) Localized surface plasmon resonance biosensing with large area of gold nanoholes fabricated by nanosphere lithography. Nanoscale Res Lett 5:818-822

10. SadAbadi H, Badilescu S, Packirisamy M, Wüthrich R (2013) Integration of gold nanoparticles in PDMS microfluidics for lab-on-a-chip plasmonic biosensing of growth hormones. Biosens Bioelectron 44:77-84

11. Park JH, Byun JY, Mun H, Shim WB, Shin YB, Li T, Kim MG (2014) A regeneratable, label-free, localized surface plasmon resonance (LSPR) aptasensor for the detection of ochratoxin A. Biosens Bioelectron 59:321-327

12. Devi RV, Doble M, Verma RS (2015) Nanomaterials for early detection of cancer biomarker with special emphasis on gold nanoparticles in immunoassays/sensors. Biosens Bioelectron 68:688-698

13. Sum TC, Bettiol AA, Van Kan JA, Watt F, Pun EYB, Tung KK (2003) Proton beam writing of low-loss polymer optical waveguides. Appl Phys Lett 83:1707-1709
14. Marcuse D (1978) Length optimization of an S-shaped transition between offset optical waveguides. Appl Opt 17:763-768

15. Remouche M, Georges F, Meyrueis P (2012) Flexible optical waveguide bent loss attenuation effects analysis and modeling application to an intrinsic optical fiber temperature sensor. Optic Photon J 2:1-7

16. Mogensen KB, El-Ali J, Wolff A, Kutter JP (2003) Integration of polymer waveguides for optical detection in microfabricated chemical analysis systems. Appl Opt 42:4072-4079

17. Liu $H$, Huang $Y$, Jiang $H$ (2016) Artificial eye for scotopic vision with bioinspired all-optical photosensitivity enhancer. Proc Natl Acad Sci USA 113:3982-3985

18. Parida OP, Bhat N (2009) Characterization of optical properties of SU-8 and fabrication of optical components. In: International conference on optics photonics. pp 4-7

19. Lin CH, Lee GB, Chen SH, Chang GL (2003) Micro capillary electrophoresis chips integrated with buried SU-8/SOG optical waveguides for bioanalytical applications. Sens Actuators A Phys 107:125-131

20. Abgrall P, Conedera V, Camon H, Gue AM, Nguyen NT (2007) SU-8 as a structural material for labs-on-chips and microelectromechanical systems. Electrophoresis 28:4539-4551

21. Borreman A, Musa S, Kok AAM, Diemeer MB, Driessen A (2002) Fabrication of polymeric multimode waveguides and devices in SU-8 photoresist using selective polymerization. In: Proceedings of the symposium IEEE/ LEOS Benelux Chapter 83-86

22. Eddings MA, Johnson MA, Gale BK (2008) Determining the optimal PDMS-PDMS bonding technique for microfluidic devices. J Micromech Microeng 18:067001

23. Hook DA, Olhausen JA, Krim J, Dugger MT (2010) Evaluation of oxygen plasma and UV ozone methods for cleaning of occluded areas in MEMS devices. J Microelectromech Syst 19:1292-1298

24. Ruano-Lopez JM, Aguirregabiria M, Tijero M, Arroyo MT, Elizalde J, Berganzo J, Aranburu I, Blanco FJ, Mayora K (2006) A new SU-8 process to integrate buried waveguides and sealed microchannels for a lab-on-achip. Sens Actuators B Chem 144:542-551

25. Schulz F, Homolka T, Bastús NG, Puntes V, Weller H, Vossmeyer T (2014) Little adjustments significantly improve the Turkevich synthesis of gold nanoparticles. Langmuir 30:10779-10784

26. Kim HM, Park JH, Jeong DH, Lee HY, Lee SK (2018) Real-time detection of prostate-specific antigens using a highly reliable fiber-optic localized surface plasmon resonance sensor combined with micro fluidic channel. Sens Actuators B Chem 273:891-898

27. Ji X, Song X, Li J, Bai Y, Yang W, Peng X (2007) Size control of gold nanocrystals in citrate reduction: the third role of citrate. J Am Chem Soc 129:13939-13948

28. Kim HM, Jeong DH, Lee HY, Park JH, Lee SK (2019) Improved stability of gold nanoparticles on the optical fiber and their application to refractive index sensor based on localized surface plasmon resonance. Opt Laser Technol 114:171-178

29. Xiao L, Jin W, Demokan MS, Ho HL, Tam HY, Ju J, Yu J (2006) Photopolymer microtips for efficient light coupling between single-mode fibers and photonic crystal fibers. Opt Lett 31:1791-1793

30. Chen J, Shi S, Su R, Qi W, Huang R, Wang M, Wang L, He Z (2015) Optimization and application of reflective LSPR optical fiber biosensors based on silver nanoparticles. Sensors 15:12205-12217

31. Mohammadi SZ, Beitollahi H, Tajik S (2018) Nonenzymatic coated screenprinted electrode for electrochemical determination of acetylcholine. Micro Nano Syst Lett 6:9

32. Sanders M, Lin Y, Wei J, Bono T, Lindquist RG (2014) An enhanced LSPR fiber-optic nanoprobe for ultrasensitive detection of protein biomarkers. Biosens Bioelectron 61:95-101

33. Kim HM, Uh M, Jeong DH, Lee HY, Park JH, Lee SK (2019) Localized surface plasmon resonance biosensor using nanopatterned gold particles on the surface of an optical fiber. Sens Actuators B Chem 280:183-191

\section{Publisher's Note}

Springer Nature remains neutral with regard to jurisdictional claims in published maps and institutional affiliations. 